\title{
The Effects of 17-Methoxyl-7-Hydroxy- Benzene-Furanchalcone on Pressure Overload-Induced Cardiac Remodeling in Rats and the Endothelial Mechanisms Based on $\mathbf{P G I}_{2}$
}

\author{
Jianchun Huang ${ }^{\mathrm{a}}$ Xiaojun Tang ${ }^{\mathrm{b}} \quad$ Xingmei Liang ${ }^{\mathrm{b}} \quad$ Qingwei Wen $^{\mathrm{a}}$ Shijun Zhang ${ }^{\mathrm{a}}$
} Feifei Xuan ${ }^{\mathrm{a}}$ Jie Jian ${ }^{\mathrm{a}}$ Xing Lin ${ }^{\mathrm{a}}$ Renbin Huang ${ }^{\mathrm{a}}$

aPharmaceutical College, Guangxi Medical University, Nanning, Guangxi, ${ }^{b}$ Department of Laboratory Medicine, Guangxi Medical College, Nanning, Guangxi, China

\section{Key Words}

MHBFC • Pressure overload $•$ Cardiac remodeling $\bullet$ Prostacyclin $\bullet$ Indomethacin

\begin{abstract}
Aim: The primary objective of this study was to study the effects of 17-methoxyl-7-hydroxybenzene-furanchalcone (MHBFC) on pressure overload-induced cardiac remodeling in rats, as well as the endothelial mechanisms based on PGI Methods: Six weeks following surgery, rats were divided randomly into the following groups: a sham group, a model group, an MHBFC $12 \mathrm{mg} / \mathrm{kg} /$ day group (MHBFC 12), an indomethacin $2 \mathrm{mg} / \mathrm{kg} /$ day group (Indo 2), and an Indo 2+ MHBFC 12 group. The MS 4000 organism signal system was used to record the rats' hemodynamic indices. Additionally, the heart weight was determined, and the cardiac remodeling index was calculated. HE and Masson's stains were utilized to perform histological analyses; the immunofluorescence was used to observe the microvessel density of myocardial tissue; the colorimetric method was used to determine the hydroxyproline content of cardiac tissue; the ELISA method was used to measure the plasma $\mathrm{PGI}_{2}$ content; and transmission electron microscopy was used to observe the ultrastructure of the myocardium. Results: A hyperdynamic circulation state, cardiac remodeling, decreased microvessel density and decreased plasma $\mathrm{PGI}_{2}$ content were each observed in the model group compared with the sham group, in which any changes in the above parameters were effectively reversed by MHBFC. Single-use Indo administration resulted in the progression of these pathophysiological changes; however, MHBFC prevented the worsening of these parameters. Conclusion: MHBFC significantly reverses pressure overload-induced cardiac remodeling, and its mechanism may partially contribute to the amelioration of endothelial cell function and the augmentation of $\mathrm{PGI}_{2}$ synthesis and secretion.
\end{abstract}

J. Huang and X.Tang contributed equally to this manuscript.

Renbin Huang

KARGER 125
Pharmaceutical College, Guangxi Medical University, 22, Shuangyong Road, Nanning, Guangxi, 530021 (The People's Republic of China)

Tel. +86-771-5339805, Fax+86/771/5358272, E-Mail huangrenbin518@163.com 


\section{Cellular Physiology Cell Physiol Biochem 2015;36:1004-1014 \begin{tabular}{l|l} 
and Biochemistry Published online: June 16, 2015 & $\begin{array}{l}\text { DO 2015 S. Karger AG, Basel } \\
\text { www.karger.com/cpb }\end{array}$ \\
\hline
\end{tabular} \\ Huang et al.: Protective Effects of MHBFC on Cardiac Remodeling}

\section{Introduction}

Hypertension is a continuum that begins with a rise in blood pressure and evolves into left ventricular hypertrophy ( $\mathrm{LVH})$, proteinuria or endothelial dysfunction; the primary pathophysiological characteristics associated with hypertension are pressure overload and cardiovascular remodeling, which includes both vascular remodeling and cardiac remodeling. If not adequately treated or controlled, remodeling may result in the development of complications, including both stroke and heart failure. Therefore, reversing pressure overloadinduced ventricular remodeling has been the focus of recent research. The development of new antihypertensive and antiremodeling drugs, which are expected to decrease the morbidity and mortality of hypertension, is the focus of this research. Endothelial dysfunction is plays an important role in both the pathogenesis and the progression of hypertensive heart disease [1-3]. The pathophysiological mechanisms of endothelial dysfunction are related to a decrease in the bioavailability of NO, as well as decreased $\mathrm{PGI}_{2}$ content and augmented ET-1 synthesis, release, and activity [4]. Several studies have determined that NSAIDs affect blood pressure in both normotensive and hypertensive individuals [5-7]. A multicenter study has demonstrated that indomethacin (Indo) attenuates the 24-hour antihypertensive effects of losartan and captopril in hypertensive patients, which may be related to decreased prostaglandin synthesis [8]. The potential of $\mathrm{PGI}_{2}$ to contribute to the beneficial effects of ACEII in the heart has been demonstrated in the attenuation of myocardial stunning and arrhythmias in the setting of ischemia-reperfusion injury [9-12], left ventricular relaxation in isolated hearts [13], the exertion of antihypertrophic effects in the setting of ANG IIinduced myocyte hypertrophy in vitro [14], the inhibition of collagen expression by $\mathrm{PGI}_{2}$ release by rat cardiac fibroblasts [15], and the reversal of pressure overload-induced cardiac remodeling in mice [16].

Millettiapulchra (Benth.) Kurz var. Laxior (Dunn) Z. Wei (Papilionaceae) (MKL) is a traditional Chinese medicinal herb distributed extensively in the Guangxi Province of China. MHBFC is a flavonoid monomer that was originally isolated from a $60 \%$ ethanol extract from MKL roots [17]. Previous studies have demonstrated that MHBFC scavenges hydroxyl radicals and oxyradicals [18], enhances the cardiocyte survival rate in the setting of hypoxia/reoxygenation injury [19], and protects the heart against myocardial ischemia both in vitro and in vivo [20], thereby preventing the pressure overload-induced progression of cardiac hypertrophy to cardiac failure by normalizing the balance between the nitric oxide signaling system and the endothelin signaling system [21]. Based on the above information, we investigated the effects of MHBFC on cardiovascular remodeling induced by pressure overload, and utilized Indo, an inhibitor of $\mathrm{PGI}_{2}$, in order to determine whether the potential mechanisms underlying the effects of remodeling are related to both the synthesis and the secretion of $\mathrm{PGI}_{2}$.

\section{Materials and Methods}

\section{Chemicals}

MHBFC (purity > 95\%) was isolated from MKL by the Department of Pharmacology, Guangxi Medical University (Guangxi, China), and characterized using UV, IR, ESI-MS, NMR, and X-ray monocrystal diffraction $[17,20]$. Indo was purchased from Shanxi YunPeng pharmaceutical Co., Ltd. All other chemicals and materials were obtained from local commercial sources.

Animals and experimental design

Male Sprague-Dawley rats weighing 130-160 g were obtained from the Guangxi Medical University Laboratory Animal Center. The rats were housed under standard conditions $\left(20-25^{\circ} \mathrm{C}, 50-60 \%\right.$ humidity, with a $12 \mathrm{~h}$ light-dark cycle) and were given standard rodent chow and free access to water. The animal experiments were approved by the Animal Ethics Committee of Guangxi Medical University and performed in accordance with the institution's guidelines. Following surgery, the rats were randomly divided into the 


\section{Cellular Physiology Cell Physiol Biochem 2015;36:1004-1014 \begin{tabular}{l|l|l}
\hline DOI: 10.1159/000430274 & (C) 2015 S. Karger AG, Basel
\end{tabular} and Biochemistry Published online: June 16, $2015 \quad$ www.karger.com/cpb \\ Huang et al.: Protective Effects of MHBFC on Cardiac Remodeling}

following groups $(n=6)$ : a sham group, in which the rats underwent a similar surgery that did not include aortic banding and were treated with distilled water; a model group, in which the rats were subjected to pressure-overload induced by abdominal aortic banding (AAB) above the renal arteries and were treated with distilled water; an MHBFC 12 group, in which the rats were treated with MHBFC at a dose of $12 \mathrm{mg} /$ $\mathrm{kg} /$ day after being subjected to $\mathrm{AAB}$; an Indo 2 group, in which the rats were treated with Indo at a dose of $2 \mathrm{mg} / \mathrm{kg} /$ day after being subjected to AAB; and an Indo $2+\mathrm{MHBFC} 12$ group, in which the rats were treated with MHBFC at a dose of $12 \mathrm{mg} / \mathrm{kg} /$ day plus Indo at a dose of $2 \mathrm{mg} / \mathrm{kg} /$ day, after being subjected to AAB. The drugs were dissolved in distilled water and were administered orally once a day for 6 weeks following surgery.

\section{General surgical procedure}

The rats were anesthetized using $10 \%$ chloral hydrate $(3 \mathrm{ml} / \mathrm{kg}$, intraperitoneal injection) and restrained in the right lateral position. Under sterile conditions, the abdominal aorta was exposed above the kidneys via a midline abdominal incision and constricted at the suprarenal level by a 4-0 silk suture tied around both the aorta and a blunted 22-gauge needle, which was then removed. The sham group was subjected to an identical treatment that omitted the abdominal aortic ligation [22, 23].

The measurement of conscious systolic blood pressure (SBP) and the heart rate (HR)

On days $0,14,28$ and 42 , both the SBPs and the HRs of all rats ( 3 times per animal, and the average was calculated) were monitored using the MS 4000 biological signal quantitative analytical system (Longfeida Technology Co., Ltd.) and the tail-cuff method, as described previously [20].

\section{The measurement of the rats' hemodynamic parameters}

On day 42, all rats were anesthetized using $10 \%$ chloral hydrate ( $3 \mathrm{ml} / \mathrm{kg}$, intraperitoneal injection), and a polyethylene catheter connected to a transducer was inserted into the right carotid artery. The relevant transducer was subsequently connected to an MS 4000 organism signal quantitative analytical system (Longfeida Technology Co., Ltd.), and the mean carotid pressures were recorded [20, 23].

After the mean carotid pressures were recorded, the polyethylene catheter was inserted into the left ventricle along the right carotid; the electrodes were plugged into the limbs for electrocardiography, and the hemodynamic parameters (including LVSP, LVEDP, $+\mathrm{dp} / \mathrm{dt}_{\max }$, and $-\mathrm{dp} / \mathrm{dt}_{\max }$ ) were recorded using the MS 4000 system for the evaluation of cardiac function [20,23].

The measurement of the cardiac remodeling indices

Following the measurements of the hemodynamic parameters, blood samples were collected from the abdominal aorta, and the rats were killed via exsanguination. The heart was promptly removed, washed with cold saline and weighed. The heart-weight index (HW/BW) was calculated by dividing the heart weight (HW) by the body weight (BW), and the left ventricular (including the interventricular septum) and right ventricular free walls were then collected separately and weighed. The left ventricular weight index (LVW/ BW) and the right ventricular weight index (RVW/BW) were then calculated.

\section{Myocardial histopathology}

The myocardial samples were fixed in neutral 10\% buffered formalin, embedded with paraffin and cut into approximately 5 -mm-thick sections, followed by both routine hematoxylin and eosin (HE) staining and Masson's staining. The myocyte cross-sectional area (in the HE stained sections, $\times 400$ ) and myocardial fibrosis (in the Masson's stained sections, $\times 100$ ) were then quantitatively analyzed using Image J $2 \times$ software. Fifty cells from the left ventricular lateral-mid free wall (including both the epicardial and the endocardial portions) were randomly selected, and the cross-sectional area of each rat was then measured. The extent of myocardial fibrosis in the tissue sections was quantitatively analyzed via morphometry in the following two ways (including the interstitial fibrosis index and the perivascular fibrosis index): first, the collagen in the myocardial interstitial spaces, excluding the perivascular areas, was visualized, and the entire area of the section was scanned. The interstitial fibrosis index was defined as the sum of the total area of collagen in the entire visual field divided by the sum of the total connective tissue area, plus the myocardial area in the entire visual field [24, 25]; second, the extent of the perivascular fibrosis of the arteries was evaluated using short-axis images of both the intramuscular arteries and the arterioles (at least 10 per rat). The area 
occupied by the artery and the area of fibrosis surrounding the artery were each traced and calculated. The perivascular fibrosis index was defined as the area occupied by the artery divided by the area of fibrosis surrounding the artery $[24,25]$. The immunofluorescence technique was used to observe the effects of MHBFC on microvessel density of hypertrophic myocardial tissue. Images from all sections were acquired using a fluorescence microscope with digital camera system. Four fields per section were randomly selected to quantify the CD34 and DAPI positive expression, the CD34 had red-staining represented the number of myocardial microvessel, the DAPI had blue-staining represented the number of myocardial cells. The myocardial microvessel density was defined as CD34 positive count/ DAPI positive count [26, 27].

\section{$\mathrm{PGI}_{2}$ and hydroxyproline measurements}

After the blood samples were collected, the heparinized blood was immediately centrifuged at $3000 \mathrm{rpm}$ for $10 \mathrm{~min}$, and all samples were stored at $-80^{\circ} \mathrm{C}$ until assayed. $\mathrm{PGI}_{2}$ was determined using a rat $\mathrm{PGI}_{2}$ ELISA kit (CUSABIO BIOTECH Co., Ltd.), according to the manufacturer's instructions. The content of hydroxyproline in the cardiac tissues was measured via a colorimetric method, using a commercial kit (Nanjing Jiancheng Bioengineering Institute, Nanjing, China).

\section{Myocardial ultrastructure}

Transmission electron microscopy (TEM, Hitachi, Japan) was used to observe the ultrastructure of the myocardium. The myocardial specimens were cut into approximately $80-\mathrm{nm}$-thick slices and then fixed in $2.5 \%$ glutaraldehyde and embedded in Epon resin. The ultrathin sections were subsequently cut and counterstained with uranyl acetate and lead citrate, and examined using a Hitachi H-7650 TEM.

\section{Statistical analysis}

All group values are presented as means \pm SDs, and the data were evaluated using the Sigma Stat (version 16.0) statistical analysis program (SPSS Inc, Chicago, IL, USA). The differences between groups were tested for statistical significance using the one-way analysis of variance (ANOVA) via the S-N-K posttest. P values less than 0.05 were considered statistically significant.

\section{Results}

The effects of MHBFC on the amelioration of the hemodynamic parameters

Following $\mathrm{AAB}$ above the renal arteries, the HR and SBP were not significantly different among the groups on day 0 , but increased significantly on days 14, 28 and 42 compared with the sham rats. These results were prevented by treatment with MHBFC for 6 weeks. Singleuse Indo resulted in additional increases in both HR and SBP in a time-dependent manner, and MHBFC significantly ameliorated these changes (Fig. 1).

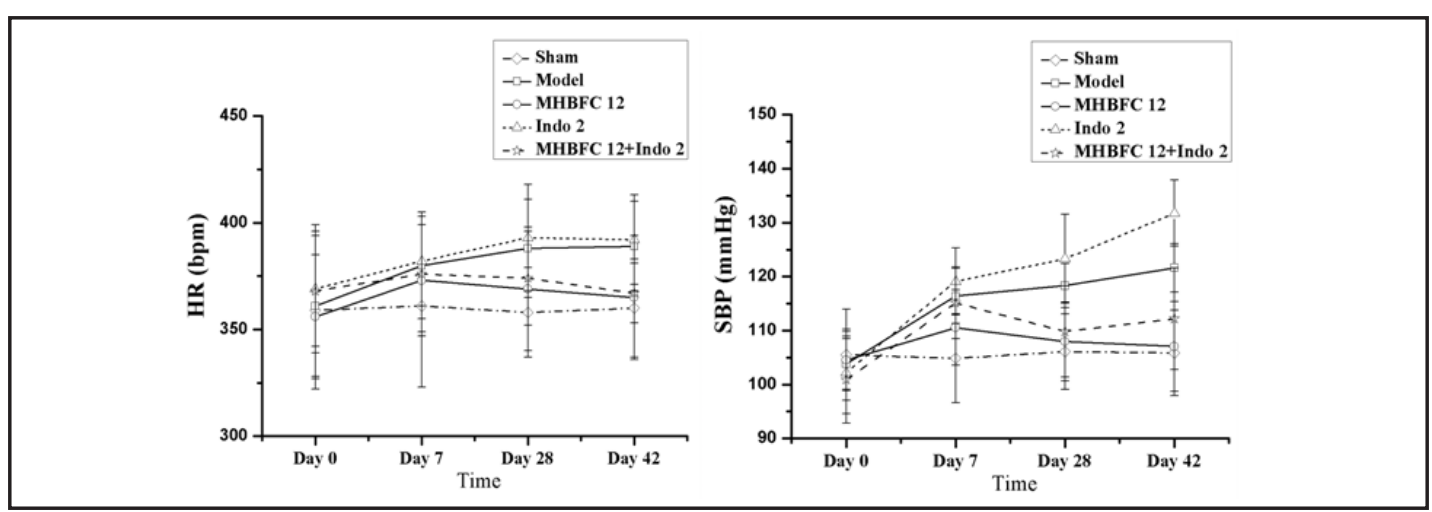

Fig. 1. The effects of Indo on the amelioration of heart rate (HR) and systolic blood pressure (SBP) by MHBFC in pressure-overloaded rats. The data are expressed as means \pm SDs, $n=6$. ${ }^{\#} P<0.05$, ${ }^{\# \#} P<0.01$ vs sham group; ${ }^{*} P<0.05,{ }^{* *} P<0.01$ vs model group; ${ }^{\triangle} P<0.05,{ }^{\triangle} \triangle P<0.01$ vs Indo 2 group. 


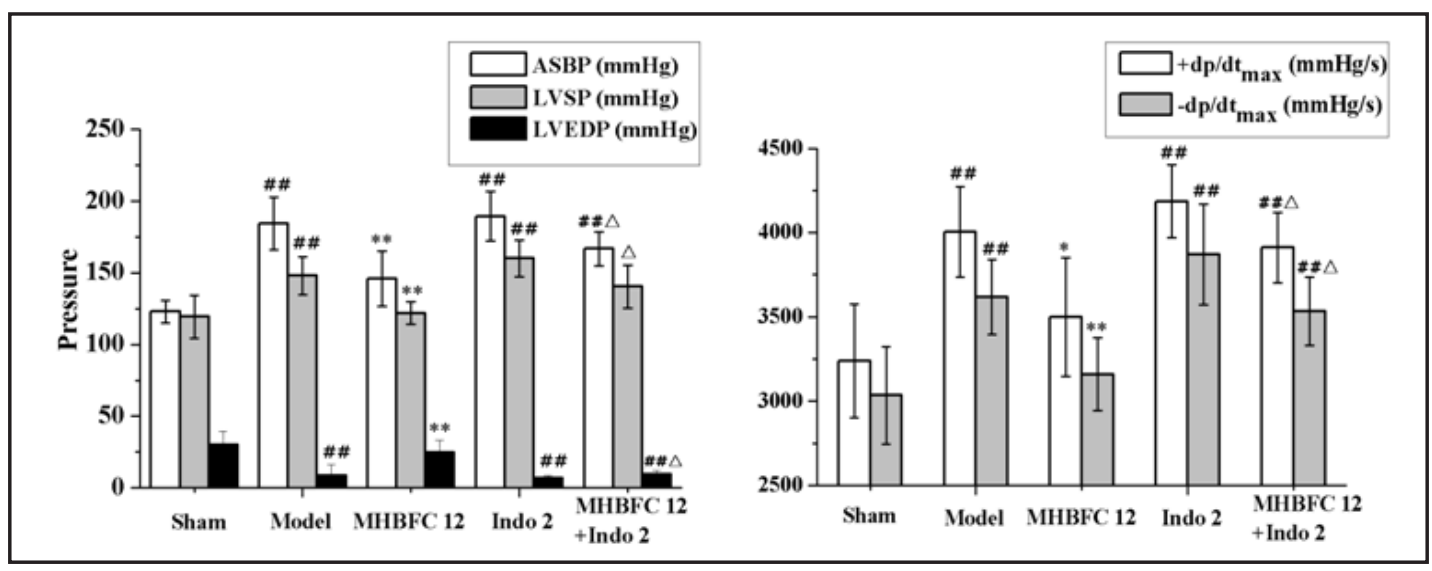

Fig. 2. The effects of Indo on the amelioration of hemodynamics, left ventricular function and body weight by MHBFC in pressure-overloaded rats. The data are expressed as means \pm SDs, $\mathrm{n}=6$. ASBP: aortic systolic blood pressure; LVSP: left ventricular systolic pressure; LVEDP: left ventricular end-diastolic pressure; $+\mathrm{dp} / \mathrm{dt}_{\text {max }}$ : maximal rate of left ventricular systolic pressure; $-\mathrm{dp} / \mathrm{dt}_{\text {max }}$ : maximal rate of left ventricular diastolic pressure. ${ }^{\#} P<0.05$, ${ }^{\# \#} P<0.01 \mathrm{vs}$ sham group; ${ }^{*} P<0.05,{ }^{* *} P<0.01$ vs model group; ${ }^{\Delta} P<0.05$, ${ }^{\triangle} P<0.01$ vs Indo 2 group.

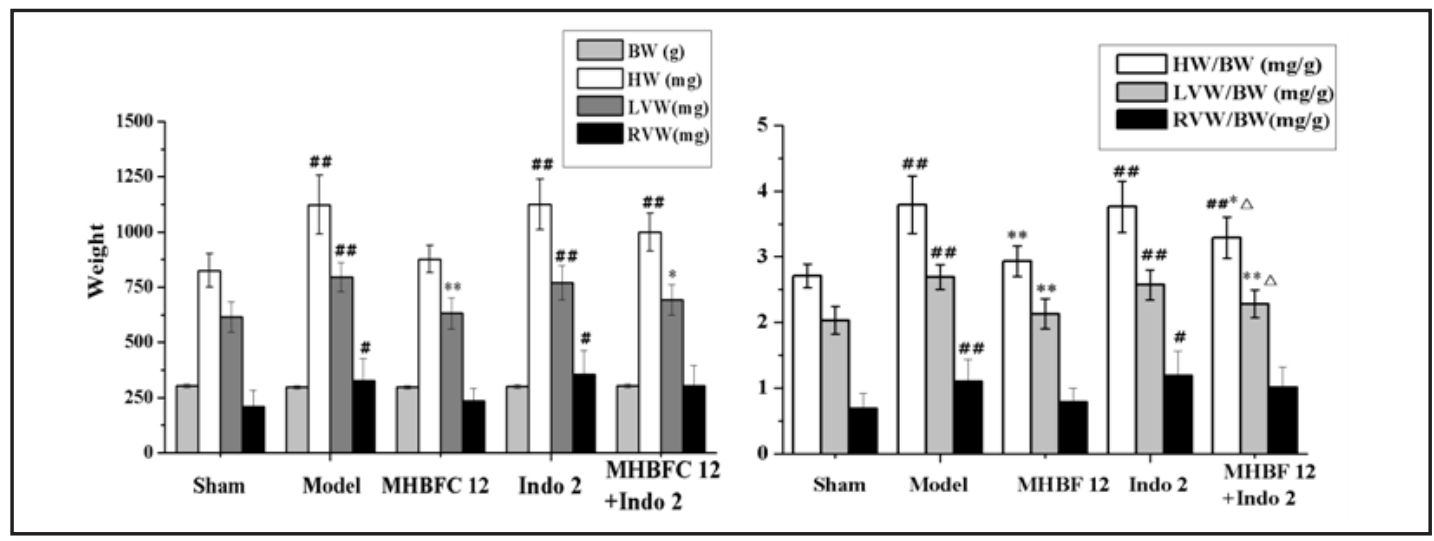

Fig. 3. The effects of Indo on the amelioration of HW, LVW, RVW, HW/BW, LVW/BW and RVW/BW by MHBFC in pressure-overloaded rats. The data are expressed as means \pm SDs, $\mathrm{n}=6$. ${ }^{\#} P<0.05,{ }^{\# \#} P<0.01$ vs sham group; ${ }^{*} P<0.05,{ }^{* *} P<0.01$ vs model group; ${ }^{\wedge} P<0.05,{ }^{\triangle} \triangle P<0.01$ vs Indo 2 group.

The right carotid aortic systolic blood pressure (ASBP) was significantly elevated in the AAB-treated rats compared with the sham-operated rats. These changes were prevented by treatment with MHBFC for 6 weeks. The ASBP in the rats in the Indo 2 group increased significantly in a time-dependent manner, and MHBFC significantly ameliorated these changes. The systolic cardiac parameters, including LVSP, $+\mathrm{d} p / \mathrm{d} t_{\text {max }}$, and the diastolic cardiac parameters, including $-\mathrm{d} p / \mathrm{d} t_{\text {max }}$, were each significantly elevated among the AAB-treated rats. By contrast, LVEDP decreased significantly. These changes were also prevented via treatment with MHBFC. Single-use Indo resulted in additional increases in cardiac contractility but reduced cardiac diastolic compliance significantly. It also inhibited the increase in cardiac contractility and ameliorated cardiac compliance in combination with MHBFC (Fig. 2).

\section{MHBFC improved pressure overload-induced left ventricular hypertrophy}

The LVH was characterized by increases in both the HW/BW and LVW/BW ratios, whereas BW was not significantly different among the groups; the HW/BW, LVW/BW, and RVW/ BW increased significantly in the Indo 2 group compared with the sham-operated controls, 
Fig. 4. The effects of MHBFC as part of the amelioration of myocardial hypertrophy in pressure-overloaded rats. The data are expressed as means \pm SDs, $n=6$. A: Representative figures of myocyte cross-section (HE stain, $\times 400$ ); B: Statistical results. ${ }^{\#} P<0.05$, ${ }^{\# \#} P<0.01$ vs sham group; ${ }^{*} P<0.05, \quad{ }^{* *} P<0.01$ vs model group; ${ }^{\triangle} P<0.05,{ }^{\triangle} \triangle P<0.01$ vs Indo 2 group.
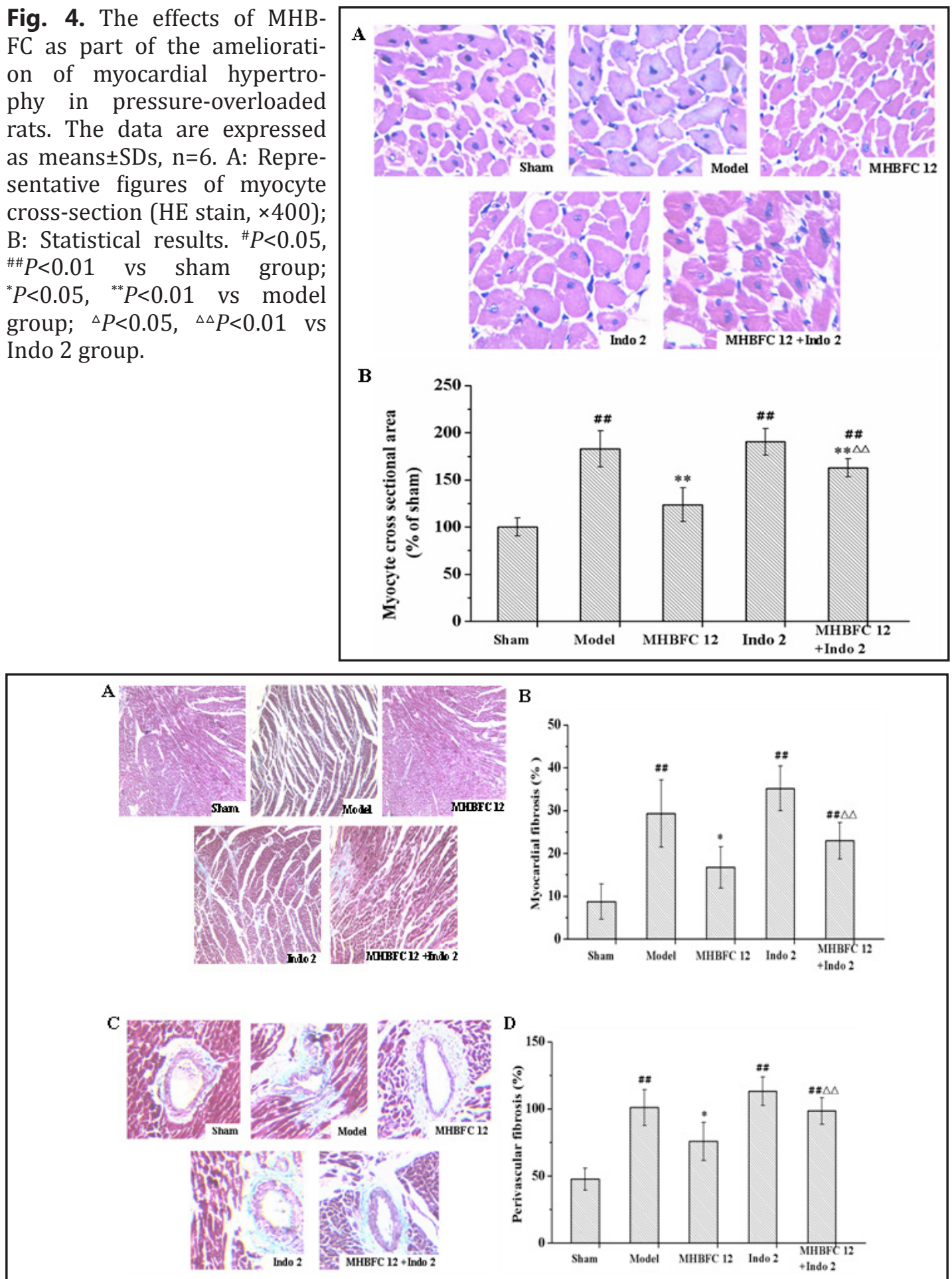

Fig. 5. The effects of MHBFC as part of the amelioration of cardiac tissue CVF and PVCA in pressure-overloaded rats. The data are expressed as means \pm SDs, $n=6$. A: Representative figures of CVF (Masson's stain, $\times 100$ ); B: Myocardial fibrosis (\%); C: Representative figures of PVCA (Masson's stain, $\times 100$ ); D: Perivascular fibrosis (\%). ${ }^{\#}<0.05,{ }^{\# \#} P<0.01$ vs sham group; ${ }^{*} P<0.05,{ }^{* *} P<0.01$ vs model group; ${ }^{\triangle} P<0.05,{ }^{\triangle} P<<0.01$ vs Indo 2 group.

and MHBFC significantly ameliorated cardiomyocyte hypertrophy (Fig. 3). The histology of the hearts from the AAB-treated rats and the rats in the Indo 2 group demonstrated that KARGER 
Fig. 6. The effects of MHBFC on microvessel density of myocardial tissue in pressure-overloaded rats. The data are expressed as means \pm SDs, $n=6$. A: Representative figures of microvascular of myocardial tissue (immunofluorescence, $\times 400$ ); B: Statistical results. ${ }^{\#} P<0.05$, ${ }^{\# \#} P<0.01$ vs sham group; ${ }^{*} P<0.05,{ }^{* *} P<0.01$ vs model group; ${ }^{\triangle} P<0.05,{ }^{\triangle} \triangle P<0.01$ vs Indo 2 group.

Fig. 7. The effects of MHBFC as part of the amelioration of cardiac tissue hydroxyproline content in pressure-overloaded rats. The data are expressed as means \pm SDs, $\mathrm{n}=6 .{ }^{*} P<0.05$, ${ }^{\# \#} P<0.01$ vs sham group; ${ }^{*} P<0.05,{ }^{* *} P<0.01$ vs model group; ${ }^{\triangle} P<0.05,{ }^{\triangle} \triangle P<0.01$ vs Indo 2 group.
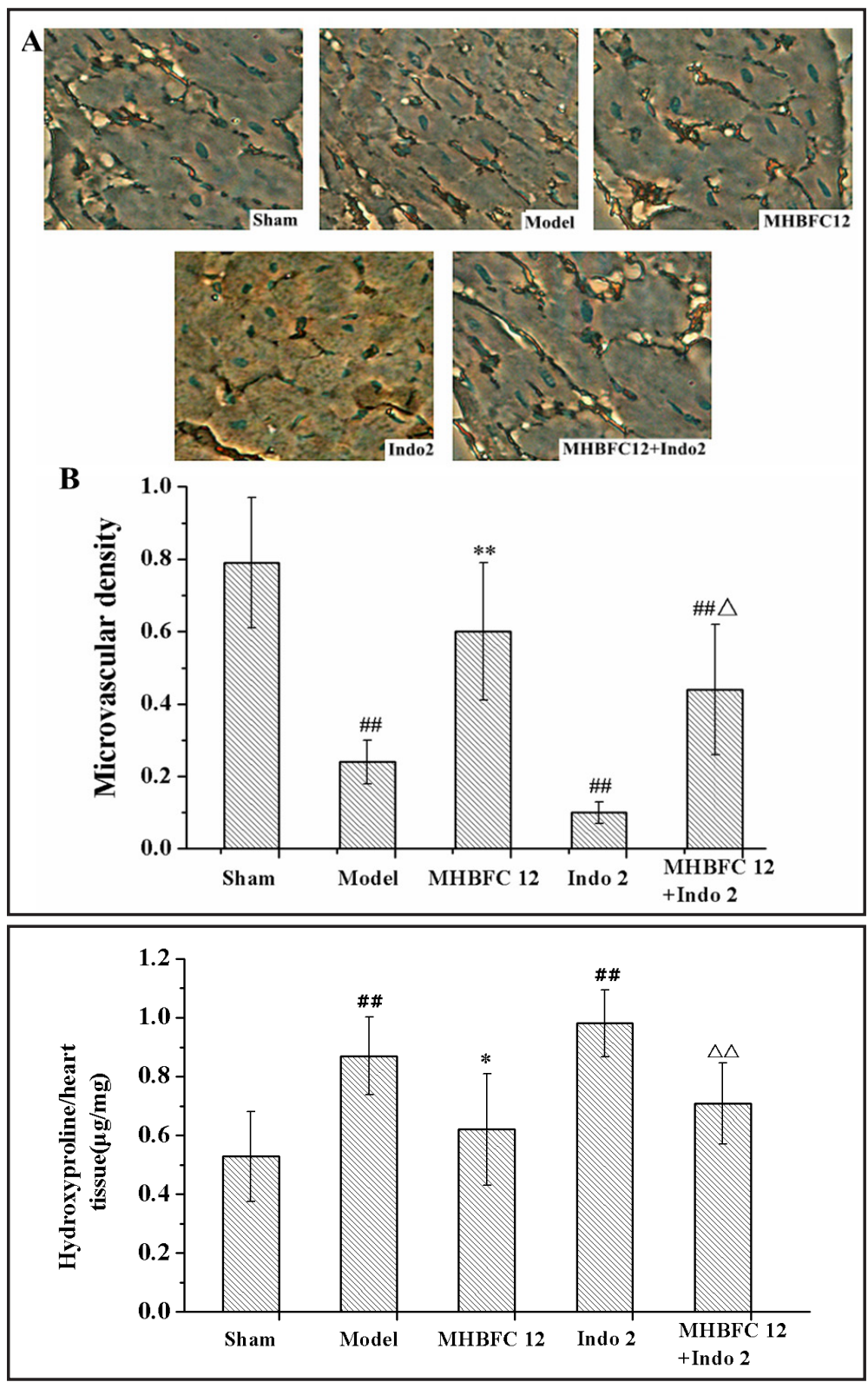

myocyte cross-sectional area increased significantly compared with the sham-operated rats, and that MHBFC significantly inhibited the increase in myocyte cross-sectional area (Fig. 4 A-B). The histology of the hearts of the AAB-treated rats demonstrated that the levels of myocardial and perivascular fibrosis both increased significantly compared with the sham group. Additionally, both the collagen volume fraction (CVF) and the perivascular collagen area (PVCA) increased significantly in the Indo 2 group, and MHBFC inhibited the decreases of both the CVF and the PVCA (Fig. 5 A-D). The microvascular density of myocardial tissue in $\mathrm{AAB}$-treated rats decreased significantly compared with the sham group, these changes were prevented via treatment with MHBFC. Single-use Indo resulted in additional decreases in microvascular density in the Indo 2 group, and MHBFC inhibited these changes (Fig. 6). Hydroxyproline content reflected the collagen levels within the cardiac tissues, and the degree of myocardial fibrosis increased in the AAB-treated rats compared with the shamoperated controls. This phenomenon was prevented via treatment with MHBFC for 6 weeks. Single-use Indo resulted in additional increases in hydroxyproline levels in the Indo 2 group, and MHBFC inhibited the increase in the level of cardiac hydroxyproline (Fig. 7). 
Fig. 8. The effects of MHBFC as part of the amelioration of plasma $\mathrm{PGI}_{2}$ content in pressure-overloaded rats. The data are expressed as means \pm SDs, $\mathrm{n}=6 .{ }^{\#} P<0.05,{ }^{\# \#} P<0.01$ vs sham group; ${ }^{*} P<0.05,{ }^{* *} P<0.01$ vs model group; ${ }^{\triangle} P<0.05,{ }^{\triangle} \triangle P<0.01$ vs Indo 2 group.

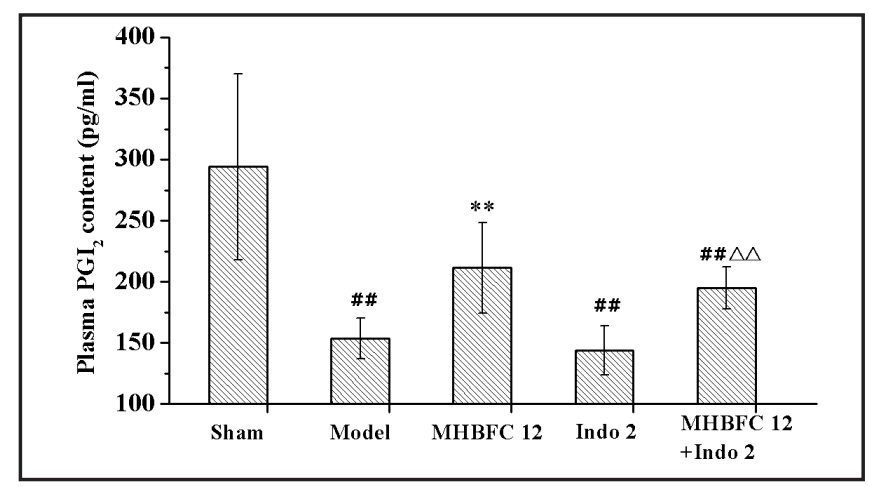

Fig. 9. Representative figures of myocardial ultrastructure among the different groups $(\times 15000)$. mf, myofibrils; $\mathrm{mi}$, mitochondria; $\mathrm{Z}$, Z-line.

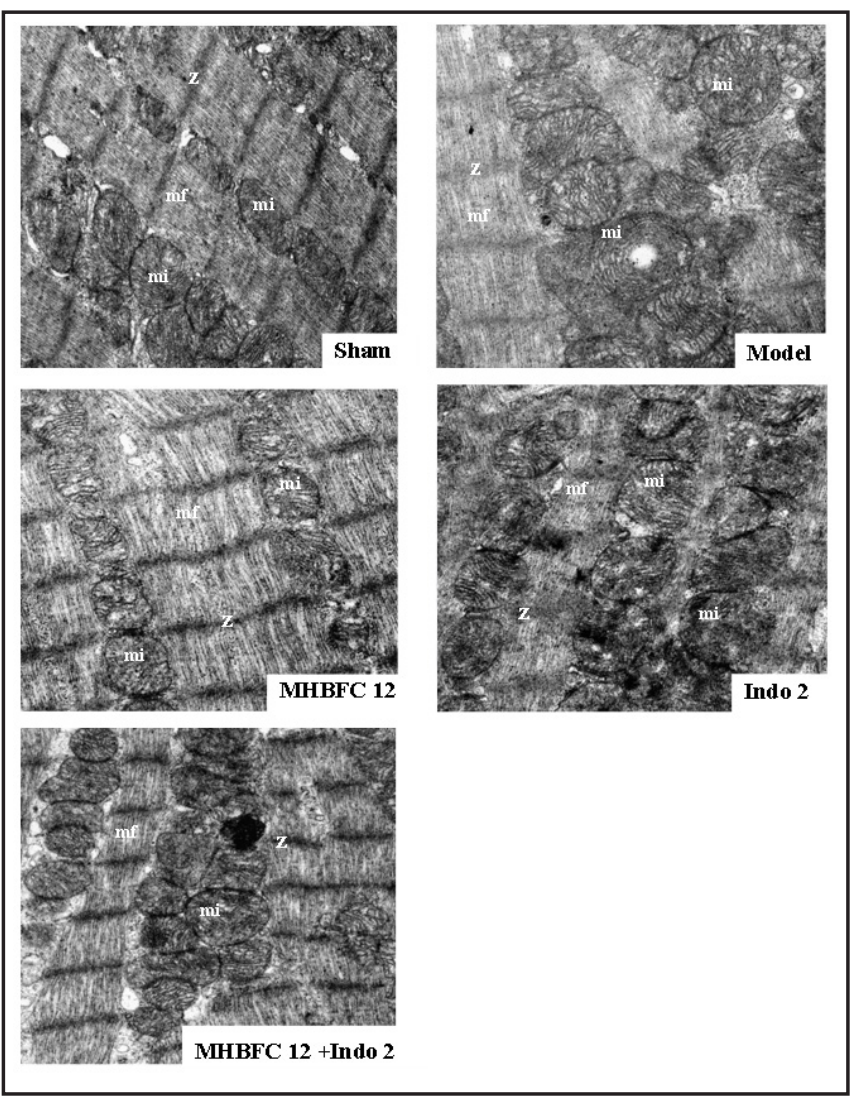

MHBFC increased plasma PGI2 content

Six weeks following aortic banding, plasma $\mathrm{PGI}_{2}$ content decreased significantly in the AAB-treated rats compared with the sham group. Long-term treatment with MHBFC significantly increased plasma $\mathrm{PGI}_{2}$ levels. The chronic administration of Indo significantly decreased plasma $\mathrm{PGI}_{2}$ content, and MHBFC inhibited the decrease in plasma $\mathrm{PGI}_{2}$ content (Fig. 8).

\section{MHBFC prevented ultrastructural alterations}

The results of an ultrastructural examination demonstrated that the ultrastructures of the cardiomyocytes in the model group rats experienced mitochondrial changes, changes characterized by diffuse mild to moderate increases in both size (hypertrophy) and number (hyperplasia), forming several rows of enlarged mitochondria that separated the myofibrils. Many of the mitochondria appeared to be both elongated as well as enlarged. Longitudinally oriented cristae, which were either parallel to the long axis of each mitochondrion, or were irregular and concentric, were observed in many areas. These features were more significant in the Indo 2 group. Following treatment with either MHBFC or Indo 2 combined with 


\section{Cellular Physiology Cell Physiol Biochem 2015;36:1004-1014 \begin{tabular}{l|l} 
and Biochemistry Published online: June 16, 2015 & $\begin{array}{l}\text { Co 2015 S. Karger AG, Basel } \\
\text { www.karger.com/cpb }\end{array}$ \\
\hline
\end{tabular} \\ Huang et al.: Protective Effects of MHBFC on Cardiac Remodeling}

MHBFC, the cardiomyocytes exhibited an almost uniform parallel myofibril arrangement. The Z-lines dividing the sarcomeres were both linear and perpendicular to the myofilaments. The myofibrils alternated with rows of ovoid mitochondria (Fig. 9).

\section{Discussion}

In this study, we used a pressure-overload model, as described previously. This AAB rat model was characterized by LVH, LV functional disorders, pulmonary congestion, and $\mathrm{RVH}$, as well as hypertension. Our previous results indicated that MHBFC prevented cardiac hypertrophy and prevented the progression of cardiac hypertrophy to cardiac failure, and that the mechanism of this action was related to the regulation of endothelial function, including the augmentation of NO release and the inhibition of the ET-1 system [21]. We continued to explore whether the potential mechanisms underlying these activities are related to the synthesis and secretion of $\mathrm{PGI}_{2}$.

$\mathrm{PGI}_{2}$, a metabolite of arachidonic acid (AA), is one of the primary prostaglandins of the cardiovascular system; its physiological activities include mediating the vasodilation of blood vessels and preventing both platelet aggregation and blood cell adhesion within the vascular wall in order to maintain blood flow, thereby preventing thrombosis and inhibiting the release of platelet thromboxane, which causes vasoconstriction. Animal experiments have demonstrated that $\mathrm{PGI}_{2}$ significantly improves myocardial ischemia/reperfusion injury in mice $[16,28]$. PGI ${ }_{2}$ has been used in the treatment of primary pulmonary hypertension, chronic obstructive pulmonary disease and acute respiratory distress syndrome. It significantly reduces pulmonary resistance, improves the ventilation/blood flow ratio, reduces right ventricular afterload, and improves ventricular function [29]. Additional studies have demonstrated that several prostaglandin receptors are expressed in the heart, including the $\mathrm{PGI}_{2}$ receptor-prostacyclin receptor (IP) $[28,29]$. The above results suggest that $\mathrm{PGI}_{2}$ plays an important role in the setting of cardiovascular disease.

In a previous animal experiment, the extent of the cardiac remodeling observed was significant in IP knock-out mice compared with wild type mice [16]. Another experiment also indicated that blood pressure, cardiac weight indices and myocardial fibrosis significantly increased in both salt-sensitive hypertensive mice and IP knock-out mice compared with wild-type mice [30]. The myocardial hypertrophy of cultured myocardial cells with the Ang II and ET-1 increased, but the participation rate of [3H]-phenylalanine was significantly decreased by Cicaprost (a PGI ${ }_{2}$ analogue) $[14,31,32]$. These results suggest that the $\mathrm{PGI}_{2}$-IP signaling pathway is involved in both ventricular remodeling and myocardial cell hypertrophy.

The results of this study demonstrated that 6 weeks following abdominal aortic banding, increases were noted in several hemodynamic parameters among the model group rats; their hearts underwent significant remodeling (cardiac weight index, myocyte crosssectional area, CVF, PVCA); microvascular density of myocardial tissue was significantly decreased; myocardial tissue hydroxyproline content was significantly elevated, and plasma $\mathrm{PGI}_{2}$ levels were decreased; however, following 6 weeks of treatment with MHBFC, these changes were ameliorated.

The results of this study also demonstrated that 6 weeks following abdominal aortic banding, increases were noted in several hemodynamic parameters among the rats in the Indo 2 group; the administration of MHBFC ameliorated these increases. Additionally, cardiac systolic and diastolic function were both significantly improved in the Indo 2 group compared with the Indo 2 group following the administration of MHBFC, as the administration of MHBFC significantly improved cardiac systolic and diastolic function, as well as the degree of myocardial fibrosis. Compared with the model group, the plasma $\mathrm{PGI}_{2}$ content of the Indo 2 group was significantly decreased; MHBFC significantly increased the level of $\mathrm{PGI}_{2}$, which was inhibited by Indo. These results suggested that $\mathrm{PGI}_{2}$ plays an important role in reversing myocardial remodeling. 


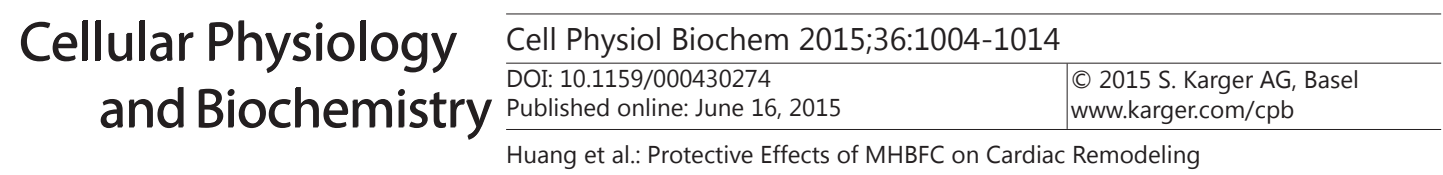

\section{Conclusions}

In conclusion, the present study demonstrated that in the setting of pressure overload, plasma $\mathrm{PGI}_{2}$ levels were significantly decreased; in the setting of endothelial dysfunction, MHBFC reversed myocardial fibrosis, improved both cardiac systolic and diastolic function, and reversed cardiac hypertrophy. Therefore, we can assume that the mechanisms that underlie its regulation of endothelial function involve the augmentation of NO release, the inhibition of the ET-1 system and the augmentation of both the synthesis and the secretion of $\mathrm{PGI}_{2}$.

\section{Disclosure Statement}

The authors declare that there are no conflicts of interest regarding the publication of this manuscript.

\section{Acknowledgments}

This project was supported by the Guangxi Scientific Research and Technology Development Research Projects (No. 0630002-2A), the Guangxi Natural Science Foundation (No. 2013GXNSFAA019175; No.2012GXNSFBA053094), and the Fund of Guangxi Key Laboratory of Functional Phytochemicals Research and Utilization (No. FPRU2013-3).

\section{References}

1 Scherrer-Crosbie M, Ullrich R, Bloch KD, Nakajima H, Nasseri B, Aretz HT, Lindsey ML, Vançon AC, Huang PL, Lee RT: Endothelial nitric oxide synthase limits left ventricular remodeling after myocardial infarction in mice. Circulation 2001;104:1286-1291.

2 Kobayashi N, Mori Y, Nakano S, Tsubokou Y, Shirataki H, Matsuoka H: Celiprolol stimulates endothelial nitric oxide synthase expression and improves myocardial remodeling in deoxycorticosterone acetate-salt hypertensive rats. J Hypertens 2001;19:795-801.

3 Casals G, Fernández-Varo G, Melgar-Lesmes P, Marfà S, Reichenbach V, Morales-Ruiz M, Jiménez W: Factors involved in extracellular matrix turnover in human derived cardiomyocytes. Cell Physiol Biochem 2013;32:1125-1136.

4 Endemann DH, Schiffrin EL: Endothelial dysfunction. J Am Soc Nephrol 2004;15:1983-1992.

5 Johnson AG, Nguyen TV, Day RO: Do nonsteroidal anti-inflammatory drugs affect blood pressure? A metaanalysis. Ann Intern Med 1994;121:289-300.

6 Houston MC: Nonsteroidal anti-inflammatory drugs and antihypertensives. Am J Med 1991;90:S42-S47.

7 Pope JE, Anderson JJ, Felson DT: A meta-analysis of the effects of nonsteroidal anti-inflammatory drugs on blood pressure. Archives of Internal Medicine 1993;153:477-484.

8 Conlin PR, Moore TJ, Swartz SL, Barr E, Gazdick L, Fletcher C, DeLucca P, Demopoulos L: Effect of indomethacin on blood pressure lowering by captopril and losartan in hypertensive patients. Hypertension 2000;36:461-465.

9 Ehring T, Baumgart D, Krajcar M, Hümmelgen M, Kompa S, Heusch G: Attenuation of myocardial stunning by the ace inhibitor ramiprilat through a signal cascade of bradykinin and prostaglandins but not nitric oxide. Circulation 1994;90:1368-1385.

10 Hartman JC: The role of bradykinin and nitric oxide in the cardioprotective action of ace inhibitors. Ann Thorac Surg 1995;60:789-792.

11 Linz W, Wiemer G, Schölkens BA: Ace-inhibition induces no-formation in cultured bovine endothelial cells and protects isolated ischemic rat hearts. J Mol Cell Cardiol 1992;24:909-919. 


\section{Cellular Physiology Cell Physiol Biochem 2015;36:1004-1014 \begin{tabular}{l|l|l}
\hline DOI: 10.1159/000430274 & C 2015 S. Karger AG, Basel
\end{tabular} www.karger.com/cpb \\ Huang et al.: Protective Effects of MHBFC on Cardiac Remodeling}

12 Liu YH, Yang XP, Sharov VG, Sigmon DH, Sabbah HN, Carretero OA: Paracrine systems in the cardioprotective effect of angiotensin-converting enzyme inhibitors on myocardial ischemia/reperfusion injury in rats. Hypertension 1996;27:7-13.

13 Anning PB, Grocott-Mason RM, Lewis MJ, Shah AM: Enhancement of left ventricular relaxation in the isolated heart by an angiotensin-converting enzyme inhibitor. Circulation 1995;92:2660-2665.

14 Ritchie RH, Schiebinger RJ, Lapointe MC, Marsh JD: Angiotensin ii-induced hypertrophy of adult rat cardiomyocytes is blocked by nitric oxide. Am J Physiol-Heart C 1998;275:H1370-H1374.

15 Yu H, Gallagher AM, Garfin PM, Printz MP: Prostacyclin release by rat cardiac fibroblasts inhibition of collagen expression. Hypertension 1997;30:1047-1053.

16 Hara A, Yuhki K, Fujino T, Yamada T, Takayama K, Kuriyama S, Takahata O, Karibe H, Okada Y, Xiao CY: Augmented cardiac hypertrophy in response to pressure overload in mice lacking the prostaglandin i2 receptor. Circulation 2005;112:84-92.

17 Jian J, Huang JC, Jao Y, Tan HD, Huang RB: Isolation and preparation of chalcone compounds from tuber of millettiapulchra var. Laxior by pre-hplc. Chin Tradit Herb Drugs 2011;42:1313-1316.

18 Jian J, Li YW, Jiang WZ, Huang RB: The effect of two chalcone monomers from yulangsan on scavenging free radicals. Chin J Gerontol 2009;29:73-74.

19 Jian J, Liu X, Huang RB, Jiang WZ: Protection and its mechanism of two flavone morphons from yulangsan on hypoxia-reoxygenation induced injury in myocardial cells. Chin Pharmacol Bullet 2009;7:942-945.

20 Jian J, Qing FZ, Zhang SJ, Huang JC, Huang RB: The effect of 17-methoxyl-7-hydroxy-benzene-furanchalcone isolated from millettiapulchra on myocardial ischemia in vitro andin vivo. Planta Med 2012;78:1324-1331.

21 Huang JC, Tang XJ, Liang XM, Wen QW, Zhang SJ, Xuan FF, Jian J, Lin X, Huang RB: The effects of 17-methoxyl-7-hydroxy-benzene-furanchalcone on the pressure overload-induced progression of cardiac hypertrophy to cardiac failure. PloS one 2014;9:e91834.

22 Doggrell S A, Brown L: Rat models of hypertension, cardiac hypertrophy and failure [J]. Cardiovasc Res1998, 39(1):89-105.

23 Gao S, Long CL, Wang RH, Wang $\mathrm{H}$ : $\mathrm{K}_{\text {ATP }}$ activation prevents progression of cardiac hypertrophy to failure induced by pressure overload via protecting endothelial function. Cardiovasc Res 2009;83: 444-456.

24 Brilla CG, Pick R, Tan LB, Janicki JS, Weber KT: Remodeling of the rat right and left ventricles in experimental hypertension. Circ Res 1990;67:1355-1364.

25 Ozono R, Matsumoto T, Shingu T,Oshima T, Teranishi Y, Kambe M, Matsuura H, Kajiyama G, Wang ZQ, Moore AF, Carey RM: Expression and localization of angiotensin subtype receptor proteins in the hypertensive rat heart. Am J Physiol Reg I 2000;278:R781-R789.

26 de Boer RA, Pinto Y M, Suurmeijer A J, Pokharel S, Scholtens E, Humler M, Saavedra JM, Boomsma F, van Gilst WH, van Veldhuisen D J: Increased expression of cardiac angiotensin II type 1 (AT1) receptors decreases myocardial microvessel density after experimental myocardial infarction. Cardiovasc Res 2003; 57:434-442.

27 Zhang JF: The effect of Qiliqiangxin capsule on microvessel injury, ventricular remodeling and metabolic remodeling in heart failure rats. PhD dissertation of Hebei Medical University, China 2013.

28 Coleman RA, Smith WL, Narumiya S: International union of pharmacology classification of prostanoid receptors: Properties, distribution, and structure of the receptors and their subtypes. Pharmacol Rev 1994;46:205-229.

29 Narumiya S, Sugimoto Y, Ushikubi F: Prostanoid receptors: Structures, properties, and functions. Physiol Rev 1999;79:1193-1226.

30 Fujino T, Nakagawa N, Yuhki K-i, Hara A, Yamada T, Takayama K, Kuriyama S, Hosoki Y, Takahata O, Taniguchi T: Decreased susceptibility to renovascular hypertension in mice lacking the prostaglandin i2 receptor ip. J Clin Invest 2004;114:805-812.

31 Ritchie RH, Rosenkranz A, Huynh L, Stephenson T, Kaye D, Dusting G: Activation of ipprostanoid receptors prevents cardiomyocyte hypertrophy via camp-dependent signaling. Am J Physiol-Heart C 2004;287:H1179-H1185.

32 Birukova AA, Zagranichnaya T, Fu P, Alekseeva E, Chen W, Jacobson JR, Birukov KG: Prostaglandins pge 2 and pgi 2 promote endothelial barrier enhancement via pka-and epac1/rap1-dependent rac activation. Exp Cell Res 2007;313:2504-2520. 